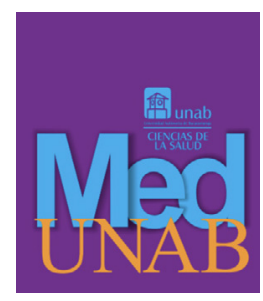

REVISTA DE LA FACULTAD

DE CIENCIAS DE LA SALUD

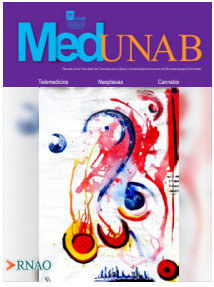

Vol. 24(2): 233-238, agosto-noviembre 2021

i-SSN $0123-7047$

Artículo de reflexión derivado de investigación

\title{
Rondas interprofesionales de heridas antes y después de Best Practice Spotlight Organization (BPSO)
}

Interprofessional wound rounds before and after becoming a Best Practice Spotlight Organization (BPSO)

Rondas interprofissionais de feridas antes e depois da Organização de Best Practice Spotlight Organization (BPSO)

\section{Patricia Skol, RN, BScN, MN, GNC (C). ${ }^{1}$ (D)}

1. Enfermera, Magíster en Enfermería y certificada en Gerontología. BPSO Sponsor at The Salvation Army Toronto Grace Health Centre, Canadá.

Correspondencia. Alma Patricia Skol. 650 Church Street, Toronto, ON M4Y 2G5, Canadá. Teléfono: 4169252251 ext.219. Email pskol@torontograce.org

\section{INFORMACIÓN DEL ARTÍCULO:}

Artículo recibido: 31 de agosto de 2020

Artículo aceptado: 22 de junio de 2021

DOI: https://doi.org/10.29375/01237047.4089

Cómo citar: Skol P. Rondas interprofesionales de heridas antes y después de Best Practice Spotlight Organization (BPSO). MedUNAB. 2021;24(2):233-238. doi: https://doi.org/10.29375/01237047.4089

\section{RESUMEN}

Introducción. El modelo de cuidado a pacientes en el Salvation Army Toronto Grace Health Centre Hospital está basado en los principios de cuidado centrado en el paciente y la familia como parte integral del equipo interprofesional. Dentro de este marco se diseñan y se implementan diferentes programas para que se mejore constantemente y se mantenga un buen nivel de cuidados y calidad de vida de los pacientes, dentro de las limitaciones que conlleva el estar hospitalizado, esto después de convertirse en Best Practice Spotlight. Objetivo. Presentar la experiencia de las rondas interprofesionales de heridas antes y después de Best Practice Spotlight. Síntesis. Antes de ser BPSO los documentos de pólizas y procedimientos que guiaban las prácticas alrededor de úlceras o heridas por presión, en el hospital se utilizaban como referencia las guías basadas en las evidencias de la Asociación de Enfermeras de Ontario (RNAO). Sin embargo, no estaba formalizada la ejecución o aplicación de estas prácticas al punto de cuidado directo al paciente. En este artículo se presenta la transición y los aspectos relevantes para que sea una realidad el cuidado centrado en el paciente y la familia como parte del equipo interprofesional en el componente de las rondas interprofesionales de heridas. Conclusiones. El éxito de la implementación efectiva 
de las guías ha sido el programa BPSO, que se ha convertido en una identidad colectiva de organizaciones que trabajan en pro de los mejores resultados en las organizaciones de salud en instituciones nacionales e internacionales

Palabras claves:

Guía de Práctica Clínica; Práctica Clínica Basada en la Evidencia; Enfermería Basada en la Evidencia; Educación en Enfermería; Cuidados de la Piel.

\begin{abstract}
Introduction. The patient care model at the Salvation Army Toronto Grace Health Centre Hospital is based on principles of patient- and family-centered care provided by the interprofessional team. In this framework, different programs are designed and implemented to constantly improve and keep a good level of care and quality of life of the patients, within the limitations involved in hospitalization, once they have become a Best Practice Spotlight. Objective. Present the experience of the interprofessional wound rounds before and after being recognized as a Best Practice Spotlight. Summary. Before becoming a BPSO, the policy and procedure documents guiding the hospital's practices on ulcers or pressure wounds used as reference the evidence-based guidelines of the Registered Nurses' Association of Ontario (RNAO). However, the implementation or application of these practices had not been formalized directly at the point of patient care. This article presents the transition and the relevant aspects for making patient- and family-centered care a reality for the interprofessional team in the component of interprofessional wound rounds. Conclusions. The success in the effective implementation of the guidelines of the BPSO program has become a collective identity for the organizations that work towards the best results in healthcare organizations in national and international institutions.
\end{abstract}

Keywords:

Clinical Practice Guideline; Evidence-Based Practice; Evidence-Based Nursing; Education, Nursing; Skin Care.

\title{
RESUMO
}

Introdução. O modelo de cuidados ao paciente no Salvation Army Toronto Grace Health Centre Hospital é baseado nos princípios de cuidados centrado no paciente e na família como parte integrante da equipe interprofissional. Dentro deste quadro, diferentes programas são concebidos e implementados para melhorar constantemente e manter um bom nível de cuidados e qualidade de vida para os pacientes, dentro das limitações de estarem hospitalizados, isto após se tornar um Best Practice Spotlight. Objetivo. Apresentar a experiência de rondas interprofissionais de feridas antes e depois do Best Practice Spotlight. Síntese. Antes de ser BPSO, a política e os documentos de procedimento que orientavam as práticas em relação às úlceras ou feridas de pressão, o hospital usava as diretrizes baseadas em evidências da Associação de Enfermeiros de Ontário (RNAO) como referência. No entanto, a execução ou aplicação dessas práticas até o atendimento direto ao paciente não estava formalizada. Este artigo apresenta a transição e aspectos relevantes para tornar uma realidade os cuidados centrados no paciente e na família como parte da equipe interprofissional no componente de rondas interprofissionais de feridas. Conclusões. O sucesso da implementação efetiva das diretrizes tem sido o programa BPSO, que se tornou uma identidade coletiva de organizações que trabalham em prol dos melhores resultados nas organizações de saúde em instituições nacionais e internacionais.

Palavras-chave:

Guia de Prática Clínica; Prática Clínica Baseada em Evidências; Enfermagem Baseada em Evidências; Educação em Enfermagem; Higiene da Pele.

\section{Introducción}

Las Guías de Práctica Clínica (GPC) refinan preguntas clínicas y mantienen un equilibrio entre los beneficios y el riesgo de una recomendación (1). Las GPC de alta calidad son redactadas por numerosas organizaciones interesadas en la calidad del cuidado con el fin de crear centros de excelencia, como es el caso de la Asociación de Enfermeras de Ontario (2). Esta asociación ha divulgado diversas guías de práctica clínica e impulsado su implementación en Canadá y otros lugares del mundo. Algunos autores manifiestan que, en general, las recomendaciones finales de una GPC van dirigidas hacia un país, una región, un hospital o una clínica. 
Estas recomendaciones pueden diferir dependiendo del grupo o región al que van dirigidas, y por estas diferencias algunos expertos prefieren no producir recomendaciones sino resúmenes de la evidencia (3)

En Ontario (Canadá) se cuenta con instituciones de salud que tienen trayectoria en la implementación de GPC, además de otras que inician en estos procesos, como el Hospital Salvation Army Toronto Grace Health Centre, ubicado en el área central de Toronto. Este es un hospital con 119 camas donde se ofrece atención médica, servicios de rehabilitación y de enfermería a pacientes con múltiples enfermedades complejas y persistentes. Se especializa en cuidado de dolencias crónicas a nivel intermedio, por lo que los pacientes pueden pasar largos periodos de tiempo bajo su cuidado. Algunos son pacientes permanentes debido a la complejidad de su diagnóstico. A pesar de permanecer clínicamente estables por largos periodos, dependen de cuidados continuos.

El modelo de cuidado a pacientes en este hospital está basado en los principios de cuidado centrado en el paciente y la familia como parte del equipo interprofesional. Así, se diseñan y se implementan diferentes programas en constante mejora con el objetivo de mantener la calidad de vida de los pacientes dentro del hospital. Como parte del esfuerzo continuo de mejoramiento en la calidad de servicios a los pacientes, el hospital se vinculó inicialmente con el programa Best Practice Spotlight Organization (BPSO) en el otoño del 2017, y oficialmente se aplicó a la Registered Nurses Association of Ontario (RNAO) para unirse y hacer parte del movimiento social BPSO (4-10).

El hospital se convirtió en una opción de organización BPSO debido de la identificación de oportunidades que mejorasen la calidad de servicios en áreas concernientes a la seguridad de sus pacientes. Colectivamente se acordó en que no se podía lograr el objetivo sin la estructura y el soporte del programa BPSO. Este artículo de reflexión busca presentar la experiencia de las rondas interprofesionales de heridas antes y después de implementar el BPSO.

\section{Antecedentes}

Parte de la preparación fue la participación activa de los empleados a todo nivel, desde el gerente ejecutivo hasta los empleados que prestan cuidado directo al paciente en diferentes niveles. Una vez que la organización RNAO aceptó al Hospital Salvation Army Toronto Grace Health Centre como pre-designado de BPSO se implementó en abril del 2018. En la actualidad está en el tercer año de las guías de práctica basadas en la evidencia.

Toronto Grace está implementando guías en áreas de prioridad de seguridad al paciente, como la prevención y manejo de heridas o úlceras por presión, evaluación y manejo del dolor, prevención de caídas y reducción de daño y cuidado centrado en la persona y la familia (1112). El impacto positivo del programa BPSO ha sido evidente a muchos niveles: no solo ha mejorado la calidad de cuidado y la seguridad de los pacientes, sino también ha aumentado el nivel de reconocimiento y apreciación por el programa BPSO por parte de los empleados, ha elevado el perfil de la organización y los equipos interdisciplinarios, y al mismo tiempo, ha establecido nuevas relaciones dentro y por fuera de la organización, lo que ha tenido un impacto muy positivo en los equipos de trabajo, los pacientes y las familias.

El hospital ha tenido un número de cambios significativos a nivel de estructura organizacional en los últimos cinco años y como resultado, está constantemente evaluando y adaptando los servicios y programas que se proveen a una comunidad de pacientes con necesidades diversas de cuidados de salud. Se ha asegurado alinear el plan de estrategia organizacional para incluir y apoyar al programa BPSO, para incrementar la capacidad dentro del equipo de liderazgo y equiparlos para que puedan facilitar y sostener este cambio en prácticas en colaboración y en consulta con los empleados de la organización, como también los pacientes, familias y amigos que hacen parte del círculo de cuidado de los pacientes (11-16).

Durante este proceso se ha tenido la oportunidad de reflexionar acerca de la organización. Se ha hecho más fuerte y ha aumentado la resiliencia a nivel individual, así como a nivel organizacional porque ha crecido y unido en comunidad con una identidad colectiva. Todo con el propósito de proveer cuidados basados en la evidencia, al mismo tiempo que se apoya y mantiene una cultura que le da prioridad a la seguridad de los pacientes haciéndolos partícipes activos del equipo interdisciplinario que se ocupa de ellos.

Se ha decidido tomar ventaja de toda oportunidad que se presenta para hacer modificaciones a las prácticas en diferentes áreas clínicas, y procesos que informan la estructura de los planes de cuidado de los pacientes. Al mismo tiempo se asegura celebrar y compartir el progreso y los resultados positivos a nivel interno y externo, compartiendo con otras organizaciones que colaboran con el hospital como parte de la implementación de programa BPSO. Estas incluyen RNAO y el Ministerio de Salud. 
Uno de los procesos que se han beneficiado de la introducción del programa BPSO en la organización, son las rondas interprofesionales de heridas. A continuación, se describe brevemente la evolución de este proceso antes y después de adoptar el programa BPSO.

\section{Rondas interprofesionales de heridas antes de BPSO}

Uno de los acercamientos más tangibles durante la implementación de las prácticas basadas en la evidencia ha sido el relacionado con la valoración y tratamiento de úlceras o heridas por presión (17-21). Durante más de 5 años, antes de 2018, se habían implementado en el hospital las rondas interprofesionales de pacientes con heridas, incluyendo heridas por presión (wound rounds). La manera en que estas rondas se llevaban a cabo no contribuyó al crecimiento profesional de las enfermeras en el hospital. Las rondas eran atendidas por enfermeras, nutricionista, fisioterapeuta, educador de enfermería y una asistente asignada a esta persona, que también era enfermera. Durante las rondas, el personal asignado al paciente participaba pasivamente y no se beneficiaba de las rondas para aprender y crecer profesionalmente $\mathrm{y}$, consecuentemente, mejorar el estado de los pacientes con diferentes tipos de heridas, incluyendo las causadas por presión. Esto explica por qué no había una transferencia efectiva de conocimiento del experto al personal de planta. Solo una persona a quien se le había asignado el título y la responsabilidad de educar había recibido la carga teórica adecuada y tenía experiencia en el área de prevención y cuidados de heridas (específicamente heridas por presión), pero su conocimiento y experiencia no fueron transferidos efectivamente a las enfermeras que ofrecían cuidados directos a pacientes, donde tendrían la oportunidad de aplicar esos conocimientos para el beneficio de los pacientes.

Todos los documentos de pólizas y procedimientos que guiaban las prácticas alrededor de úlceras o heridas por presión en el hospital utilizaban como referencia las guías basadas en la evidencia de RNAO. Pero no estaba formalizada la ejecución o aplicación de estas prácticas al punto de cuidado directo al paciente.

\section{Rondas Interprofesionales de Heridas: Después de BPSO}

Una vez que se formalizó el programa BPSO con RNAO en la primavera de 2018, se inició la implementación de las guías basadas en la evidencia de RNAO. Se inició la implementación con la primera, que fue la Guía de valoración, manejo y prevención de heridas por presión. Se condujo el análisis para identificar qué recomendaciones se necesitarían para mejorar las prácticas en esta área clínica.

Como parte de este análisis también se llevaron a cabo auditorías de las historias clínicas de los pacientes con heridas, para ver si las enfermeras estaban documentando apropiadamente la información requerida de acuerdo a las guías basadas en la evidencia y también para confirmar si se estaban utilizando las herramientas recomendadas de acuerdo a las guía de RNAO, como estaba previamente estipulado en las pólizas del hospital, para valorar las heridas y determinar objetivamente si los pacientes estaban mejorando.

Los resultados de las auditorías revelaron que solamente la persona que estaba en la posición de educador de enfermeras y su asistente habían documentado la totalidad de la información de evolución clínica y tratamientos recomendados para todos los pacientes que tenían heridas por presión. Esta práctica se mantuvo por un periodo de varios años. Cuando se entrevistó a las enfermeras que estaban encargadas del cuidado directo a los pacientes, se identificó que ninguna había valorado directamente la herida como parte de la ronda interprofesional y que dejaban todas las decisiones en manos de la personal a cargo de educación de las enfermeras y su asistente.

El impacto negativo de esta práctica fue evidente. Las enfermeras casi en su totalidad, con la excepción de algunos que practicaban en otros hospitales, no habían adquirido el conocimiento y la experiencia necesaria para prestar atención óptima a este grupo de pacientes. Aunque una situación alarmante, al mismo tiempo esto representó una oportunidad ideal para implementar sistemáticamente la guía relacionada con el área de cuidado de heridas por presión, basada en la evidencia con la estructura de BPSO.

Se estableció el equipo BPSO con líderes campeones (champions) representando varias profesiones, no solo enfermería, en todas las áreas del hospital. Se seleccionó una unidad piloto para iniciar la implementación de la guía, con la idea de expandirla más adelante al resto del hospital. Se proporcionó educación a las enfermeras en el área de valoración y manejo de las heridas por presión, de acuerdo con las recomendaciones de la guía RNAO. Una vez que se completó la educación, las enfermeras y ahora los pacientes y sus familias que deseaban estar activamente envueltos, fueron invitados a participar en el diseño de las nuevas rondas 
de pacientes con heridas, donde tanto las enfermeras como los pacientes y sus familias, si deseaban tomar parte, comenzaron a tener una participación activa y dinámica durante las rondas. El personal de enfermería empezó a documentar los resultados de la valoración de las heridas en la historia clínica de los pacientes. Esto se determinó por observación directa durante las rondas y también con auditoría de las historias clínicas que revelaron resultados positivos, indicando que hubo transferencia de conocimiento a la práctica clínica.

\section{BPSO: Transformando la práctica de enfermería con evidencia y conocimiento}

El modelo para transferir conocimiento a la práctica o práctica en acción, Knowledge To Action (KTA), es el modelo recomendado por RNAO para la implementación de las guías basadas en la evidencia y la transferencia de conocimientos de la evidencia a la práctica. KTA tiene componentes fundamentales que facilitan la adopción, retención y aplicación de conceptos y prácticas relevantes de parte de las enfermeras y otros profesionales (15-18). En este modelo se tienen en cuenta aspectos como la evidencia, el contexto y la facilitación de la transferencia de conocimientos. Estos elementos están interactuando constantemente en un ciclo dinámico que incluye evaluación continua para adaptar la evidencia al contexto a medida que este evoluciona y cambia.

El cambio positivo que se observa en la práctica de las enfermeras en este escenario clínico se puede atribuir a la implementación sistemática de la guía para valoración y el manejo de heridas por presión de la RNAO y a la estructura que proporciona el programa BPSO. Un aspecto fundamental de la implementación de las guías es la aplicación del modelo KTA.

Antes de ser una organización pre-designada como BPSO no se tenía estructura ni modelo que rigiera la implementación de las guías basadas en la evidencia. Esto a pesar de que sí se tenía acceso a estas guías, en teoría, desde que RNAO las hace disponibles a quienes las deseen. Pero el éxito de la implementación efectiva de las guías ha sido el programa BPSO. Más que un programa, como BPSO hace parte de una identidad colectiva de organizaciones que trabajan con otras encargadas de programas de salud a nivel nacional e internacional, que sistemáticamente seleccionan y utilizan prácticas basadas en la evidencia para cambiar y mejorar la calidad de servicios de salud y la calidad de vida de pacientes y comunidades en todo el mundo.

\section{Conclusiones}

The Salvation Army Toronto Grace Health Centre se siente orgulloso y privilegiado de ser parte del movimiento social BPSO y con entusiasmo y dedicación se continúa progresando en la implementación de las guías basadas en la evidencia de RNAO. Se espera compartir y celebrar con la comunidad el éxito una vez se dé cumplimiento a los tres años de implementación de las guías, es decir en el año 2021 y ser oficialmente nombrado como una organización designada BPSO. Esto debido al desarrollo de una identidad colectiva con las organizaciones encargadas de los programas de salud a través de la organización RNAO en Canadá y en otras regiones del mundo.

\section{Referencias}

1. Cortés OL, Serna-Restrepo A, Salazar-Beltrán LD, Rojas-Castañeda YA, Cabrera-González S, ArévaloSandoval I. Implementación de guías de práctica clínica de la Asociación de Enfermeras de OntarioRNAO: Una experiencia de enfermería en un hospital colombiano. MedUNAB. 2016;19(2):103-14. https:// doi.org/10.29375/01237047.2617

2. Bajnok I, Grinspun D, Lloyd M, McConnel H. Liderando mejoras de calidad mediante el desarrollo, implementación, y medición de las guías de buenas prácticas de Enfermería. MedUNAB, 2015;17(3):155162. https://doi.org/10.29375/01237047.2382

3. Silva GA. Implementación de guías de buenas prácticas clónicas elaboradas por Registered Nurses Association of Ontario en Curriculum de Enfermería Universidad de Chile. MedUNAB. 2015;17(3):182189. https://doi.org/10.29375/01237047.2386

4. Aznar AS, Gonzalez CD. Organizational staff training: Profiling organizations to predict training efficiency. J Intellect Disabil Res. 2012;56(7-8):778.

5. Baker R, Camoso-Stefinovic J, Gillies C, Shaw EJ, Cheater F, Flottorp S, et al. Tailored interventions to overcome identified barriers to change: Effects on professional practice and health care outcomes. Cochrane Database Syst Rev. 2010;3:CD005470. https://pubmed.ncbi.nlm.nih.gov/20238340/

6. Blue AV. Nursing leadership in an era of collaboration. En: Glazer G, Fitzpatrick J. Nursing leadership from the outside in. 1era. ed. New York: Springer Publishing Co; 2013. p.21-30.

7. Brodbeck FC, Kerschreiter R, Mojzisch A, Frey $\mathrm{D}$, Schulz-Hardt S. The dissemination of critical, unshared information in decision-making groups: the effects of pre-discussion dissent. Eur J Soc Psychol. 2002;32(1):35-56. https://doi.org/10.1002/ejsp.74 
8. Brouwers M, Stacey D, O'Connor A. Knowledge translation tools. In Straus S, Tetroe J, Graham ID, editors. Knowledge translation in healthcare: Moving from evidence to practice. 1st ed. Hoboken, NJ: Wiley-Blackwell; 2009, 50-62 p.

9. Buchanan DA, Fitzgertald L, Ketley D. The sustainability and spread of organizational change. 1st ed. New York: Routledge; 2007. 336 p. https://doi. org/10.4324/9780203030653

10. Burgers JS, Cluzeau, FA, Hanna SE, Hunt C, Grol R. Charactieristics of high-quality guidelines: Evaluation of 86 clinical guidelines developed in ten European countries and Canada. International Journal of Technological Assessment in Health Care. 2003;19(1):148-157. https://doi.org/10.1017/ S026646230300014X

11. Chang EH, Doucette WR. Factors influencing interprofessional collaboration: A literature review from 2004-2011. Value in Health. 2012;15(7):A622-A623. https://doi.org/10.1016/j. jval.2012.08.125

12. Grinspun D, McConell H. Forging the way with implementation science. En: Grinspun D, Bajnok I (Eds.). Transforming Nursing Through Knowledge. Best Practices for Guideline Development, Implementation Science, and Evaluation. 1era. Ed. Indianapolis, IN, USA: Sigma Theta Tau International Honor Society of Nursing; 2018. pp.96-116.

13. Canadian Foundation for Healthcare Improvement. Job Satisfaction and Retention of Nursing Staff: The Impact of Nurse Management Leadership [Internet]. Canadian Health Services Research Foundation; 2006. Disponible en: http://www.cfhi-fcass.ca/Migrated/ PDF/ResearchReports/OGC/gagnon 1325 e.pdf.

14. Canadian Nurses Association Website. Nursing Care Delivery Models [Internet]. Canadian Nurses Association; 2009. Disponible en: https:/www.cnaaiic.ca/ /media/cna/page-content/pdf-en/nursing care delivery models_e.pdf
15. David D, Davis N. Selecting educational interventions for knowledge translation. CMAJ Open. 2010;182:89-93. https://doi.org/10.1503/ cmaj.081241

16. Davies B, Edwards N. Sustaining knowledge use. In Straus S, Tetroe J, Graham ID, editors. Knowledge translation in healthcare: Moving from evidence to practice. 1st ed. Hoboken, NJ: WileyBlackwell; 2009. p. 165-73.

17. Grinspun D. Health policy in changing environments. In Taples E, Ray S, Hannon R (editors). In Canadian perspectives on advanced nursing practice: Clinical practice, research, leadership, consultation and collaboration (1st Canadian ed.). Toronto, ON: Canadian Scholar's Press; 2016. p. 285-300.

18. Straus S, Tetroe J, Graham ID. Defining knowledge translation. CMAJ Open. 2009;181(3-4):165-168. https://doi.org/10.1503/cmaj.081229

19. Dobbins M, Davies B, Danseco E, Edwards N, Virani T. Changing nursing practice: Evaluating the usefulness of a best-practice guideline implementation Toolkit. Can J Nurs Leadersh. 2005;18(1):34-35. https://doi.org/10.12927/ cjnl.2005.17034

20. Edwards N, Rowan M, Marck P, Grinspun D. Understanding whole systems change in health care: the case of nurse practitioners in Canada. Policy Polit Nurs Pract. 2011;12(1):4-17. https:// doi.org/10.1177/1527154411403816

21. Knowles M. The Adult Learner: A Neglected Species. 2nd ed. Houston, TX: Gulf Publishing Company; 1978. Chapter 3, The emergence of a theory of adult learning: andragogy; p.27-59. 